\title{
On new traveling wave solutions of the Hirota-Satsuma coupled KdV equation
}

\author{
M. Tuncay Gencoglu ${ }^{1}$, Ali Akgul ${ }^{2}$ and Mustafa Inc ${ }^{3}$ \\ ${ }^{1}$ Firat University, Vocational School of Technical Sciences, Elazıg, Turkey \\ ${ }^{2}$ Siirt University, Art and Science Faculty, Depatment of Mathematics, Siirt,Turkey \\ ${ }^{3}$ Firat University, Science Faculty, Depatment of Mathematics,23119 Elazg, Turkey
}

Received: 22 February 2017, Accepted: 24 April 2017

Published online: 26 August 2017.

\begin{abstract}
The improved ( $\left.\mathrm{G}^{\prime} / \mathrm{G}\right)$-expansion method is applied to reach the different type soliton solitions of the Hirota-Satsuma Coupled $\mathrm{KdV}(\mathrm{HSCKdV})$ equation. It is obtained hyperbolic, triangular, periodic wave and kink soliton solitions of this equation. The method is an effective one to reach the different types of solutions of nonlinear partial differential equations and systems. Finally, the numerical simulations add to these obtained solutions.
\end{abstract}

Keywords: Improved ( $\left.\mathrm{G}^{\prime} / \mathrm{G}\right)$-expansion method, Hirota-Satsuma coupled KdV equation, soliton.

\section{Introduction}

The mathematical modeling of scientific systems generally is expressed by nonlinear evolution equations. Therefore, it is crucial to reach general solutions of these corresponding nonlinear equations. Thus, the general solutions of these equations provide much information about the character and the structure of the equations for researchers. Many effective methods have been improved to provide much information for physicians and engineers. We recall that most of these methods use the wave variable transformation to reduce the nonlinear PDE to ODE in order to acquire the solution. Several are tanh [1], G'/G-expansion [2], Jacobi elliptic function [3], mapping [4], tanh-sech [5],exp-function [6] and first integral methods [7]. All of these methods are effective methods for acquiring traveling wave solutions NPDE. For more details see $[8,9,10]$.

The improved $\left(\mathrm{G}^{\prime} / \mathrm{G}\right)$-expansion method has been presented to the literature by Liu et al.[7]. This method has been successfully implemented to NPDE and some fractional differential equations which are new type of equations. The aim of this paper is to find travelling wave solutions of the HSCKdV equation by using improved $\left(\mathrm{G}^{\prime} / \mathrm{G}\right)$-expansion method.

This paper is organized as follows: the HSCKdV equation is given in Section 2 and improved $\left(\mathrm{G}^{\prime} / \mathrm{G}\right)$-expansion method is described in Section 3. This method has been applied to the HSCKdV equation [6] in Section 4. Then we give some conclusions in the last Section. 


\section{Mathematical analysis}

In this work, we consider the generalized HSCKdV equation [6]

$$
\begin{aligned}
u_{t} & =\frac{1}{2} u_{x x x}-3 u u_{x}+3(v w)_{x} \\
v_{t} & =-v_{x x x}+3 u v_{x} \\
w_{t} & =-w_{x x x}+3 u w_{x}
\end{aligned}
$$

where the subscripts $t$ and $x$ denote differention. With $w=v^{*}$ and $w=v$, Eq.(1) reduces, respectively, to a new complex coupled KdV equation [6] and Hirota-Satsuma equation [12].

Eq. (1) has been studied to obtain analitical and numerical solutions by many authors. Such as, numerically, variational iteration method [13],two-dimensional differential transform method [14,15], Adomian's method [16], homotopy analysis method [17]. Analitically, improve Riccati equation method [18], fractional sub-equation method [19], homogeneous balance method [20], etc.

\section{Improved $\left(G^{\prime} / G\right)$-expansion method}

In this method, we assume that

$$
u(\xi)=\sum_{i=0}^{m} a_{i} F_{i}(\xi)
$$

is solution function such that

$$
F(\xi)=\frac{G^{l}(\xi)}{G(\xi)}
$$

where $G(\xi)$ is given by

$$
G(\xi) G^{\prime \prime}(\xi)=A G^{2}(\xi)+B G(\xi) G^{\prime}(\xi)+C\left(G^{\prime}(\xi)\right)^{2}
$$

$G(\xi)$ is the solution of the second-order nonlinear diferential equation such that $A, B, C$ are real parameters. If we try to find the solution of the (3), then we find the following four cases [7].

Case 1: If $B \neq 0, \Delta=B^{2}+4 A-4 A C \geq 0$, then

$$
F(\xi)=\frac{B}{2(1-c)}+\frac{B \sqrt{\Delta}}{2(1-c)} \frac{c_{1} e^{\frac{\sqrt{\Delta}}{2} \xi}+c_{2} e^{\frac{\sqrt{\Delta}}{2} \xi}}{c_{1} e^{\frac{\sqrt{\Delta}}{2} \xi}+c_{2} e^{\frac{\sqrt{\Delta}}{2} \xi}}
$$

such that $c_{1}, c_{2}$ are real parameters.

Case 2: If $B \neq 0$ and $\Delta=B^{2}+4 A-4 A C<0$, then

$$
F(\xi)=\frac{B}{2(1-c)}+\frac{B \sqrt{-\Delta}}{2(1-c)} \frac{i c_{1} \cos \frac{\sqrt{-\Delta}}{2} \xi-i c_{2} \sin \frac{\sqrt{-\Delta}}{2} \xi}{i c_{1} \sin \frac{\sqrt{-\Delta}}{2} \xi+i c_{2} \cos \frac{\sqrt{-\Delta}}{2} \xi}
$$

Case 3: If $B=0$ and $\Delta=A(c-1) \geq 0$, then

$$
F(\xi)=\frac{\sqrt{\Delta}}{(1-c)} \frac{c_{1} \cos (\sqrt{\Delta}) \xi+c_{2} \sin (\sqrt{\Delta}) \xi}{c_{1} \sin (\sqrt{\Delta}) \xi-c_{2} \cos (\sqrt{\Delta}) \xi} .
$$


Case 4: If $B=0$ and $\Delta=A(c-1)<0$, then

$$
F(\xi)=\frac{\sqrt{\Delta}}{(1-c)} \frac{i c_{1} \cosh (\sqrt{-\Delta}) \xi-c_{2} \sinh (\sqrt{-\Delta}) \xi}{i c_{1} \sinh (\sqrt{-\Delta}) \xi-c_{2} \cosh (\sqrt{-\Delta}) \xi} .
$$

Let apply the presented improved $\left(G^{\prime} / G\right)$-expansion method to Hirota-Satsuma coupled KdV equation. If we use

$$
u(x, t)=u(\xi), \quad v(x, t)=v(\xi), \quad w(x, t)=w(\xi), \quad \xi=x-k t
$$

transformations in

$$
\begin{aligned}
u_{t} & =\frac{1}{2} u_{x x x}-3 u u_{x}+3(v w)_{x} \\
v_{t} & =-v_{x x x}+3 u v_{x} \\
w_{t} & =-w_{x x x}+3 u w_{x}
\end{aligned}
$$

such that $k$ is wave velocity, then we get

$$
\left\{\begin{array}{l}
-k u^{\prime}-\frac{1}{2} u^{\prime \prime \prime}+3 u u^{\prime}-3(v w)^{\prime}=0 \\
-k v^{\prime}+v^{\prime \prime \prime}-3 u v^{\prime}=0 \\
-k w^{\prime}+w^{\prime \prime \prime}-3 u w^{\prime}=0
\end{array}\right.
$$

We can take

$$
m_{1}=2, \quad m_{2}=m_{3}=1
$$

by order balancing because of the higher order linear and nonlinear terms in (3) [21]. Thus for (9)

$$
\left\{\begin{array}{l}
u(\xi)=a_{0}+a_{1} F(\xi)+a_{2} F^{2}(\xi) \\
v(\xi)=b_{0}+b_{1} F(\xi) \\
w(\xi)=c_{0}+c_{1} F(\xi)
\end{array}\right.
$$

such a solution can be searched, where $a_{0}, a_{1}, a_{2}, b_{0}, b_{1}, c_{0}, c_{1}$ are constants that will be determined and $F(\xi)$ is the solution function of the (3). If we take necessary derivatives in (10) and put in (9), then the following algebraic equation system is obtained.

$$
\begin{aligned}
\left(\frac{G^{\prime}}{G}\right)^{0}: & A^{2} a_{1}+3 A a_{0} a_{1}-3 A^{2} a_{2} B-\frac{1}{2} A a_{1} B^{2}-A^{2} a_{1} C-3 A b_{1} c_{0}-3 A b_{0} c_{1}-A a_{1} k=0 \\
\left(\frac{G^{\prime}}{G}\right)^{1}: & A^{2} a_{1}^{2}+8 A^{2} a_{2}+6 A a_{0} a_{2}+4 A a_{1} B+3 a_{0} a_{1} B-7 A a_{2} B^{2}-\frac{1}{2} a_{1} B^{3}-8 A^{2} a_{2} C \\
& -4 A a_{1} B C-3 B b_{1} c_{0}-3 B b_{0} c_{1}-6 A b_{1} c_{1}-2 A a_{2} k-a_{1} B k=0 \\
\left(\frac{G^{\prime}}{G}\right)^{2}: & -4 A a_{1}-3 a_{0} a_{1}+9 A a_{1} a_{2}+3 a_{1}^{2} B+26 A a_{2} B+6 a_{0} a_{2} B+\frac{7}{2} a_{1} B^{2}-4 a_{2} B^{3}+8 A a_{1} C+3 a_{0} a_{1} C-26 A a_{2} B C \\
& -\frac{7}{2} D_{1} B^{2} C-4 A a_{1} C^{2}+3 b_{1} c_{0}-3 b_{1} C c_{0}+3 b_{0} c_{1}-6 B b_{1} c_{1}-3 b_{0} C c_{1}+a_{1} k-2 a_{2} B k-a_{1} C k=0 \\
\left(\frac{G^{\prime}}{G}\right)^{3}: & -3 a_{1}^{2}-20 A a_{2}-6 a_{0} a_{2}+6 A a_{2}^{2}-6 a_{1} B+9 a_{1} a_{2} B+19 a_{2} B^{2}+3 a_{1}^{2} C+40 A a_{2} C \\
& +6 a_{0} a_{2} C+12 a_{1} B C-19 a_{2} B^{2} C-20 A a_{2} C^{2}-6 a_{1} B C^{2}+6 b_{1} c_{1}-6 b_{1} C c_{1}+2 a_{2} k-2 a_{2} C k=0
\end{aligned}
$$




$$
\begin{aligned}
& \left(\frac{G^{\prime}}{G}\right)^{4}: 3 a_{1}-9 a_{1} a_{2}-27 A^{2} B+6 a_{2}^{2} B-9 a_{1} C+9 a_{1} a_{2} C+54 a_{2} B C+9 a_{1} C^{2}-27 a_{2} B C^{2}-3 a_{1} C^{3}=0 \\
& \left(\frac{G^{\prime}}{G}\right)^{5}: 12 a_{2}-6 a_{2}^{2}-36 a_{2} C+6 a_{2}^{2} C+36 a_{2} C^{2}-12 a_{2} C^{3}=0 \\
& \left(\frac{G^{\prime}}{G}\right)^{0}:-2 A^{2} b_{1}-3 A a_{0 b_{1}}+A B^{2} b_{1}+2 A^{2} b_{1} C-A b_{1} k=0 \\
& \left(\frac{G^{\prime}}{G}\right)^{1}:-3 A a_{1} b_{1}-8 A B b_{1}-3 a_{0} B b_{1}+B^{3} b_{1}+8 A B b_{1} C-B b_{1} k=0 \\
& \left(\frac{G^{\prime}}{G}\right)^{2}: 8 A b_{1}+3 a_{0} b_{1}-3 A a_{2} b_{1}-3 a_{1} B b_{1}-7 B^{2} b_{1}-16 A b_{1} C-3 a_{0} b_{1} C+7 B^{2} b_{1} C+8 A b_{1} C^{2} \\
& +b_{1} k-16 A b_{1} C-3 a_{0} b_{1} C+7 B^{2} b_{1} C+8 A b_{1} C^{2}+b_{1} k-b_{1} C k=0 \\
& \left(\frac{G^{\prime}}{G}\right)^{3}: 3 a_{1} b_{1}+12 B b_{1}-3 a_{2} B b_{1}-3 a_{1} b_{1} C-24 B b_{1} C+12 B b_{1} C^{2}=0 \\
& \left(\frac{G^{\prime}}{G}\right)^{4}:-6 b_{1}+3 a_{2} b_{1}+18 b_{1} C-3 a_{2} b_{1} C-18 b_{1} C^{2}+6 b_{1} C^{3}=0 \\
& \left(\frac{G^{\prime}}{G}\right)^{0}:-2 A^{2} c_{1}-3 A a_{0} c_{1}+A B^{2} c_{1}+2 A^{2} C c_{1}-A c_{1} k=0 \\
& \left(\frac{G^{\prime}}{G}\right)^{1}:-3 A a_{1} c_{1}-8 A B c_{1}-3 a_{0} B c_{1}+B^{3} c_{1}+8 A B C c_{1}-B c_{1} k=0 \\
& \left(\frac{G^{\prime}}{G}\right)^{2}: 8 A c_{1}+3 a_{0} c_{1}-3 A a_{2} c_{1}-3 a_{1} B c_{1}-7 B^{2} c_{1}-16 A C c_{1}-3 a_{0} C c_{1} \\
& +7 B^{2} C c_{1}+8 A C^{2} c_{1}+c_{1} k-C c_{1} k=0 \\
& \left(\frac{G^{\prime}}{G}\right)^{3}: 3 a_{1} c_{1}+12 B c_{1}-3 a_{2} B c_{1}-3 a_{1} C c_{1}-24 B C c_{1}+12 B C^{2} c_{1}=0 \\
& \left(\frac{G^{\prime}}{G}\right)^{4}:-6 c_{1}+3 a_{2} c_{1}+18 C c_{1}-3 a_{2} C c_{1}-18 C^{2} c_{1}+6 C^{3} c_{1}=0 .
\end{aligned}
$$

If we solve this algebraic equation system by Mathematica, then we acquire the desired constants as

$$
\begin{aligned}
& B=0 \\
& a_{0}=\frac{1}{3}\left(-2 A+B^{2}+2 A C-k\right), \quad a_{1}=0, \quad a_{2}=2\left(1-2 C+C^{2}\right) \\
& b_{0}=0, \quad b_{1} \neq 0 \\
& c_{0}=0, c_{1}=\frac{4(1-C)^{2}(A(1-C)+k)}{3 b_{1}}
\end{aligned}
$$

and

$$
\begin{aligned}
& B \neq 0 \\
& a_{0}=\frac{1}{3}(-2 A+2 A C-k), \quad a_{1}=2(-B+B C), \quad a_{2}=2\left(1-2 C+C^{2}\right) \\
& b_{0}=0, b_{1} \neq 0 \\
& c_{0}=\frac{(1-C) B\left(-B^{2}-4 A(1-C)+4 k\right)}{3 b_{1}}, \quad c_{1}=\frac{-c_{0}+C c_{0}}{B} .
\end{aligned}
$$

If these constants are put in (3.10), then the complete solutions of Hirota-Satsuma coupled KdV equation are given by 


\section{Case 1:}

$$
\begin{gathered}
u(x, t)=\frac{1}{3}(-2 A(1-C)-k)-\frac{B^{2}}{2}\left[1-\Delta\left(\frac{c_{1} e^{\frac{\sqrt{\Delta}}{2} \xi}+c_{2} e^{-\frac{\sqrt{\Delta}}{2} \xi}}{c_{1} e^{\frac{\sqrt{\Delta}}{2} \xi}-c_{2} e^{-\frac{\sqrt{\Delta}}{2} \xi}}\right)^{2}\right] \\
v(x, t)=\frac{b_{1} B}{2(1-C)}\left[1+\sqrt{\Delta}\left(\frac{c_{1} e^{\frac{\sqrt{\Delta}}{2} \xi}+c_{2} e^{-\frac{\sqrt{\Delta}}{2} \xi}}{c_{1} e^{\frac{\sqrt{\Delta}}{2} \xi}-c_{2} e^{-\frac{\sqrt{\Delta}}{2} \xi}}\right)\right], \quad b_{1} \neq 0
\end{gathered}
$$

and

$$
w(x, t)=\frac{B(1-C)\left(-B^{2}-4 A(1-C)+4 k\right)}{6 b_{1}}\left[1-\sqrt{\Delta}\left(\frac{c_{1} e^{\frac{\sqrt{\Delta}}{2} \xi}+c_{2} e^{-\frac{\sqrt{\Delta}}{2} \xi}}{c_{1} e^{\frac{\sqrt{\Delta}}{2} \xi}-c_{2} e^{-\frac{\sqrt{\Delta}}{2} \xi}}\right)\right] .
$$

If we take $-c_{1}=c_{2}$ in (13)-(15), then we will obtain the following soliton solutions as

$$
\begin{gathered}
u(x, t)=\frac{1}{3}(-2 A(1-C)-k)-\frac{B^{2}}{2}\left[1-\Delta \tanh ^{2} \frac{\sqrt{\Delta}}{2} \xi\right] \\
v(x, t)=\frac{b_{1} B}{2(1-C)}\left[1+\sqrt{\Delta} \tanh \frac{\sqrt{\Delta}}{2} \xi\right]
\end{gathered}
$$

and

$$
w(x, t)=\frac{B(1-C)\left(-B^{2}-4 A(1-C)+4 k\right)}{6 b_{1}}\left[1-\sqrt{\Delta} \tanh \frac{\sqrt{\Delta}}{2} \xi\right] .
$$

If we use

$$
\tanh ^{2} y=1-\sec h^{2} y
$$

in Eq. (16), then we get another single wave solution as

$$
u(x, t)=\frac{1}{3}(-2 A(1-C)-k)+\frac{B^{2}}{2}(\Delta-1)-\frac{B^{2}}{2} \Delta \sec h^{2} \frac{\sqrt{\Delta}}{2} \xi .
$$

If we take $c_{1}=c_{2}$ in (13)-(15), then we acquire

$$
\begin{gathered}
u(x, t)=\frac{1}{3}(-2 A(1-C)-k)-\frac{B^{2}}{2}\left[1-\Delta \operatorname{coth}^{2} \frac{\sqrt{\Delta}}{2} \xi\right] \\
v(x, t)=\frac{b_{1} B}{2(1-C)}\left[1+\sqrt{\Delta} \operatorname{coth} \frac{\sqrt{\Delta}}{2} \xi\right]
\end{gathered}
$$

and

$$
w(x, t)=\frac{B(1-C)\left(-B^{2}-4 A(1-C)+4 k\right)}{6 b_{1}}\left[1-\sqrt{\Delta} \operatorname{coth} \frac{\sqrt{\Delta}}{2} \xi\right] .
$$

Case 2:

$$
u(x, t)=\left(\frac{1}{3}-2 A(1-C)-k\right)-\frac{B^{2}}{2}\left[1+\Delta\left[\frac{i c_{1} \cos \frac{\sqrt{-\Delta}}{2} \xi-c_{2} \sin \frac{\sqrt{-\Delta}}{2} \xi}{i c_{1} \sin \frac{\sqrt{-\Delta}}{2} \xi+c_{2} \cos \frac{\sqrt{-\Delta}}{2} \xi}\right]^{2}\right]
$$




$$
v(x, t)=\frac{b_{1} B}{2(1-C)}\left[1+\sqrt{-\Delta} \frac{i c_{1} \cos \frac{\sqrt{-\Delta}}{2} \xi-c_{2} \sin \frac{\sqrt{-\Delta}}{2} \xi}{i c_{1} \sin \frac{\sqrt{-\Delta}}{2} \xi+c_{2} \cos \frac{\sqrt{-\Delta}}{2} \xi}\right], b_{1} \neq 0
$$

and

$$
w(x, t)=\frac{B(1-C)\left(-B^{2}-4 A(1-C)+4 k\right)}{6 b_{1}}\left[1-\sqrt{-\Delta} \frac{i c_{1} \cos \frac{\sqrt{-\Delta}}{2} \xi-c_{2} \sin \frac{\sqrt{-\Delta}}{2} \xi}{i c_{1} \sin \frac{\sqrt{-\Delta}}{2} \xi+c_{2} \cos \frac{\sqrt{-\Delta}}{2} \xi}\right] .
$$

Case 3:

$$
\begin{gathered}
u(x, t)=\frac{1}{3}\left(-2 A(1-C)+B^{2}-k\right)+2 \Delta\left[\frac{c_{1} \cos (\sqrt{\Delta} \xi)+c_{2} \sin (\sqrt{\Delta} \xi)}{c_{1} \sin (\sqrt{\Delta} \xi)-c_{2} \cos (\sqrt{\Delta} \xi)}\right]^{2} \\
v(x, t)=\frac{b_{1} \sqrt{\Delta}}{(1-C)}\left[\frac{c_{1} \cos (\sqrt{\Delta} \xi)+c_{2} \sin (\sqrt{\Delta} \xi)}{c_{1} \sin (\sqrt{\Delta} \xi)-c_{2} \cos (\sqrt{\Delta} \xi)}\right], b_{1} \neq 0
\end{gathered}
$$

and

$$
w(x, t)=\frac{4(1-C)(A(1-C)+k)}{3 b_{1}}\left[\sqrt{\Delta} \frac{c_{1} \cos (\sqrt{\Delta} \xi)+c_{2} \sin (\sqrt{\Delta} \xi)}{c_{1} \sin (\sqrt{\Delta} \xi)-c_{2} \cos (\sqrt{\Delta} \xi)}\right] .
$$

Case 4:

$$
\begin{gathered}
u(x, t)=\frac{1}{3}\left(-2 A(1-C)+B^{2}-k\right)-2 \Delta\left[\frac{i c_{1} \cosh (\sqrt{-\Delta} \xi)-c_{2} \sinh (\sqrt{-\Delta} \xi)}{i c_{1} \sinh (\sqrt{-\Delta} \xi)-c_{2} \cosh (\sqrt{-\Delta} \xi)}\right]^{2}, \\
v(x, t)=\frac{b_{1} \sqrt{\Delta}}{(1-C)}\left[\frac{i c_{1} \cosh (\sqrt{-\Delta} \xi)-c_{2} \sinh (\sqrt{-\Delta} \xi)}{i c_{1} \sinh (\sqrt{-\Delta} \xi)-c_{2} \cosh (\sqrt{-\Delta} \xi)}\right], \quad b_{1} \neq 0
\end{gathered}
$$

and

$$
w(x, t)=\frac{4(1-C)(A(1-C)+k)}{3 b_{1}}\left[\sqrt{-\Delta} \frac{i c_{1} \cosh (\sqrt{-\Delta} \xi)-c_{2} \sinh (\sqrt{-\Delta} \xi)}{i c_{1} \sinh (\sqrt{-\Delta} \xi)-c_{2} \cosh (\sqrt{-\Delta} \xi)}\right] .
$$

If we take $c_{1}=0, c_{2} \neq 0$ and $c_{1} \neq 0, c_{2}=0$ in (23)-(25) respectively, then we acquire

$$
\begin{gathered}
u_{1}(x, t)=\frac{1}{3}-2 A(1-C)-k-\frac{B^{2}}{2}\left[1-\Delta \tan \frac{\sqrt{-\Delta}}{2}(x-k t)\right]^{2} \\
u_{2}(x, t)=\frac{1}{3}-2 A(1-C)-k-\frac{B^{2}}{2}\left[1+\Delta \cot \frac{\sqrt{-\Delta}}{2}(x-k t)\right]^{2} \\
v_{1}(x, t)=\frac{b_{1} B}{2(1-C)}\left[1-\sqrt{-\Delta} \tan \frac{\sqrt{-\Delta}}{2}(x-k t)\right], b_{1} \neq 0 . \\
v_{2}(x, t)=\frac{b_{1} B}{2(1-C)}\left[1+\sqrt{-\Delta} \cot \frac{\sqrt{-\Delta}}{2}(x-k t)\right], b_{1} \neq 0 \\
w_{1}(x, t)=\frac{B(1-C)\left(-B^{2}-4 A(1-C)+4 k\right)}{6 b_{1}}\left[1+\sqrt{-\Delta} \tan \frac{\sqrt{-\Delta}}{2}(x-k t)\right]
\end{gathered}
$$

and

$$
w_{2}(x, t)=\frac{B(1-C)\left(-B^{2}-4 A(1-C)+4 k\right)}{6 b_{1}}\left[1-\sqrt{-\Delta} \cot \frac{\sqrt{-\Delta}}{2}(x-k t)\right] \text {. }
$$


If we take $c_{1}=0, \quad c_{2} \neq 0$ and $c_{1} \neq 0, \quad c_{2}=0$ in (26)-(28) respectively, then we get

$$
\begin{gathered}
u_{3}(x, t)=\frac{1}{3}\left(-2 A(1-C)+B^{2}-k\right)-2 \Delta \tan ^{2}[\sqrt{\Delta}(x-k t)] \\
u_{4}(x, t)=\frac{1}{3}\left(-2 A(1-C)+B^{2}-k\right)+2 \Delta \cot ^{2}[\sqrt{\Delta}(x-k t)] \\
v_{3}(x, t)=-\frac{b_{1} \sqrt{\Delta}}{(1-C)} \tan [\sqrt{\Delta}(x-k t)], b_{1} \neq 0 \\
v_{4}(x, t)=\frac{b_{1} \sqrt{\Delta}}{(1-C)} \cot [\sqrt{\Delta}(x-k t)], b_{1} \neq 0 \\
w_{3}(x, t)=-\frac{4(1-C)(A(1-C)+k)}{3 b_{1}} \sqrt{\Delta} \tan [\sqrt{\Delta}(x-k t)]
\end{gathered}
$$

and

$$
w_{4}(x, t)=\frac{4(1-C)(A(1-C)+k)}{3 b_{1}} \cot [\sqrt{\Delta}(x-k t)] .
$$

If we take $c_{1}=0, \quad c_{2} \neq 0$ and $c_{1} \neq 0, \quad c_{2}=0$ in (3.28)-(3.30) respectively, then we obtain

$$
\begin{gathered}
u_{5}(x, t)=\frac{1}{3}\left(-2 A(1-C)+B^{2}-k\right)-2 \Delta \tanh ^{2}[\sqrt{-\Delta}(x-k t)], \\
u_{6}(x, t)=\frac{1}{3}\left(-2 A(1-C)+B^{2}-k\right)-2 \Delta \operatorname{coth}^{2}[\sqrt{-\Delta}(x-k t)] \\
v_{5}(x, t)=\frac{b_{1} \sqrt{\Delta}}{(1-C)} \tanh [\sqrt{-\Delta}(x-k t)], b_{1} \neq 0, \\
v_{6}(x, t)=\frac{b_{1} \sqrt{\Delta}}{(1-C)} \operatorname{coth}[\sqrt{-\Delta}(x-k t)], b_{1} \neq 0, \\
w_{5}(x, t)=\frac{4(1-C)(A(1-C)+k)}{3 b_{1}} \sqrt{-\Delta}, \tanh [\sqrt{-\Delta}(x-k t)]
\end{gathered}
$$

and

$$
w_{6}(x, t)=\frac{4(1-C)(A(1-C)+k)}{3 b_{1}} \sqrt{-\Delta} \operatorname{coth}[\sqrt{-\Delta}(x-k t)] .
$$

Analytic solutions of Hirota-Satsuma coupled KdV equation (HSCKdV) given by (8) were investigated by improved $\left(\frac{G^{I}}{G}\right)$-expansion method presented by Liu at al. [7], in this work. We obtained complex, single wave, soliton, trigonometric and singular solutions of (8) by this method. In addition, we showed that these solutions ensure the system (8) by Mathematica. HSCKdV equation has been solved by many authors by using various methods. HSCKdV equation was solved by Feng and Li [21] by using Fan sub equation method with balancing terms $m=2$ and $m=1$. Feng ve Zhang [22] acquired jacobi elliptic solutions of HSCKdV equation by auxiliary function method. Yan [23] found wave solutions of HSCKdV equation by using

$$
u=\alpha v^{2}+\beta v+\gamma, w=A v+B,
$$

transformations by extended Jacobi elliptic function method such that $\alpha, \beta, \gamma, A$ and $B$ are constants that will be determined. We obtained some of the solitons that have been found by the methods that we mentioned and also we found different solutions in this work. 


\section{Numerical simulations}

We will discuss the physical properties of the results of the results of the HSCKdV equation. Some obtained solitions are shown in Figures 1-6. These figures have the following physical comments.

The shapes of Eqs. (13)-(15) and (16)-(18) are represented in Figs.1-6. Eq. (13) is singular solitary wave solution. In Fig. 1 both 3D and 2D graphs describe the singular solitary wave solution, the wave speed is $k=0.05$ within $-4 \leq x, t \leq 4$. Eq. (14) is singular king solution and is given Fig.2 In Fig.2,both 3D and 2D graphs describe the behaviour of $v(x, t)$, the wave speed is $k=0.1$ within $-10 \leq x, t, \leq 10$. Eq. (15) is singular kink solution and is given Fig.3. Eq. (16) is non-topological soliton solutions and are given Figs. 5 and 6, respectively.

Results of this work are new soliton solutions of HSCKdV equation. The results are in terms of hyperbolic, triangular and exponential functions, and hence they all produce topological solutions (16)-(18), bell-shaped solitary wave solutions (19)-(21), periodic wave solutions (32)-(33) and similar waves. These solutions are useful in study shallow water waves. The integrability of NLPDEs can be studied by using the improved $\left(G^{\prime} / G\right)$-expansion method. The obtained soliton solutions of this study are going to be useful for interested in the HSCKdV equation.
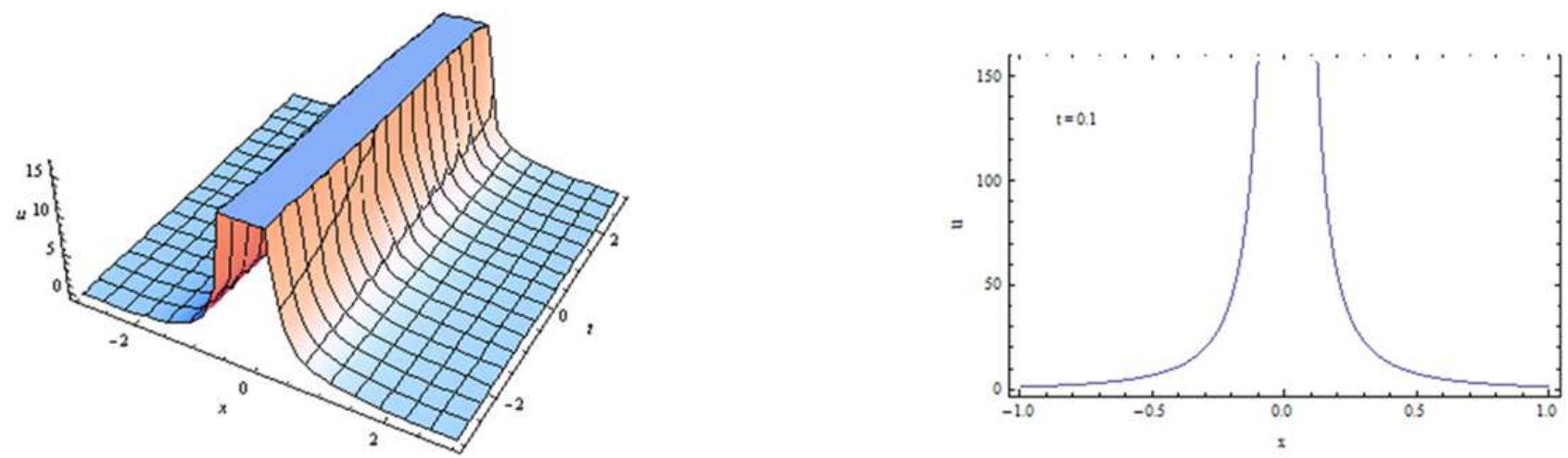

Fig. 1: Three dimensional and two dimensional graphics of the soliton solution of $u(x, t)$ that given by (13) such that $A=2, B=C=1, k=0.05, c_{1}=c_{2}=0.1$.
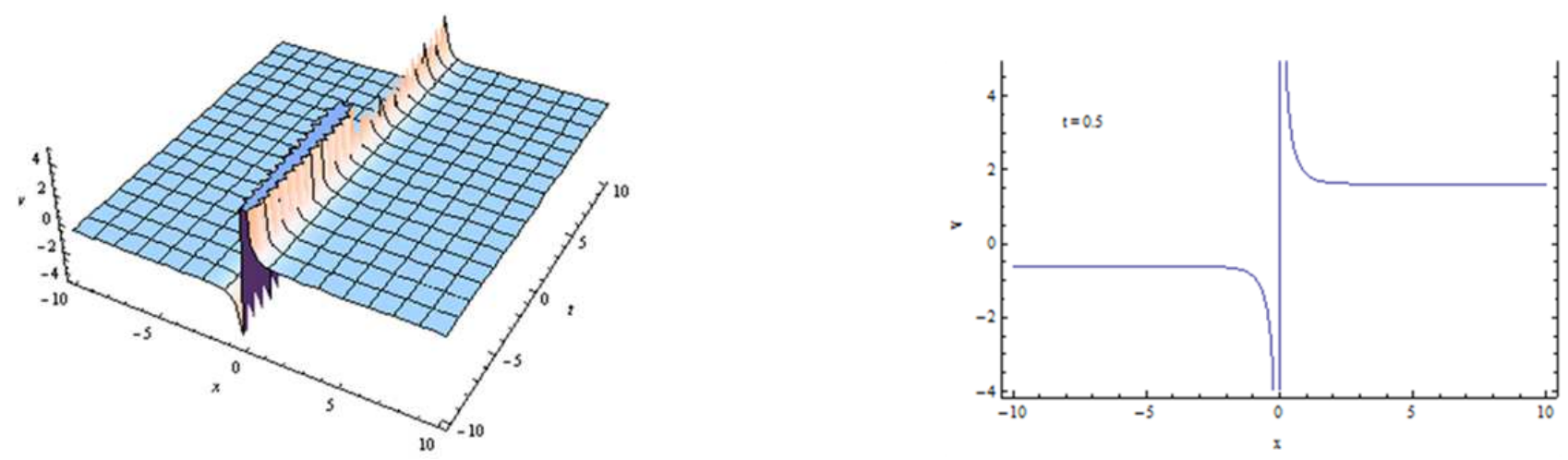

Fig. 2: Three dimensional and two dimensional graphics of soliton solution of $v(x, t)$ that given by (14) such that $A=2$, $B=1, C=0.5, k=0.01, c_{1}=c_{2}=0.1, b_{1}=0.5$. 

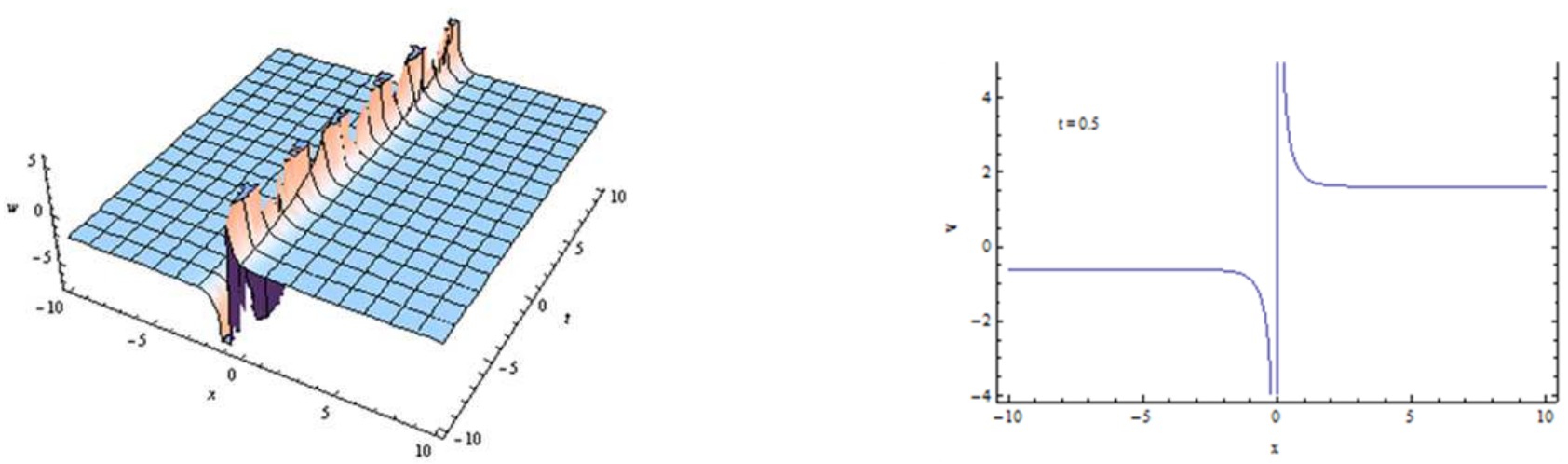

Fig. 3: Three dimensional and two dimensional graphics of soliton solution of $w(x, t)$ that given by (15) such that $A=2$, $B=1, C=0.5, k=0.1, c_{1}=c_{2}=0.1, b_{1}=0.5$.
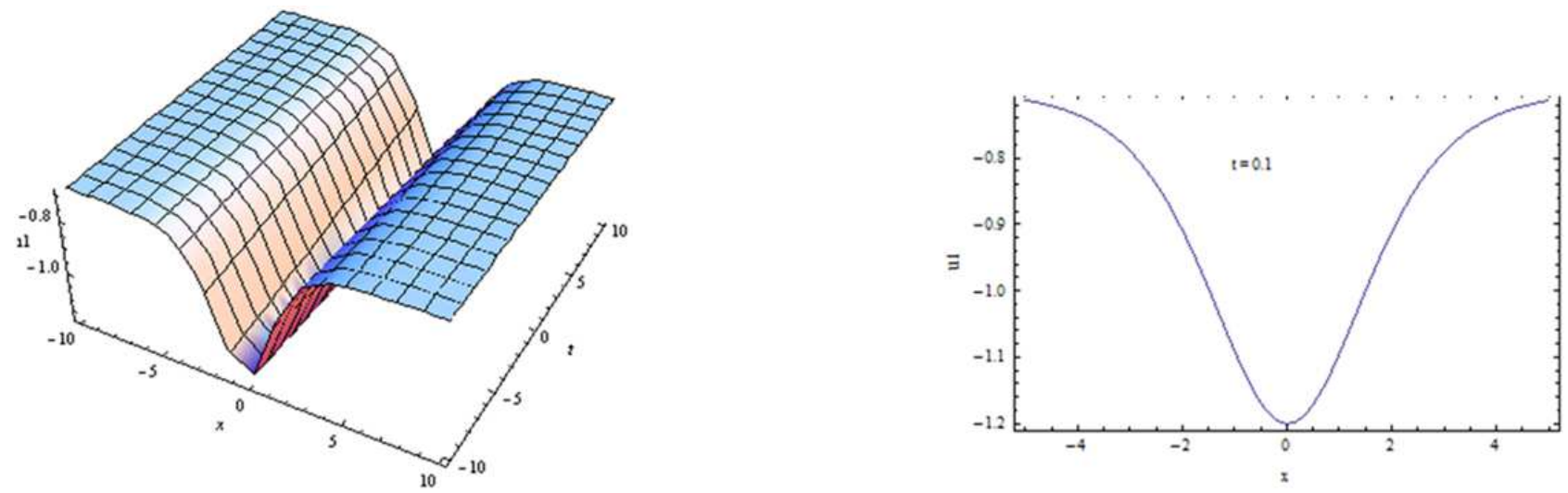

Fig. 4: Three dimensional and two dimensional graphics of the soliton solution of $u(x, t)$ that given by (16) such that $A=2, B=C=1, k=0.05, c_{1}=c_{2}=0.1$.
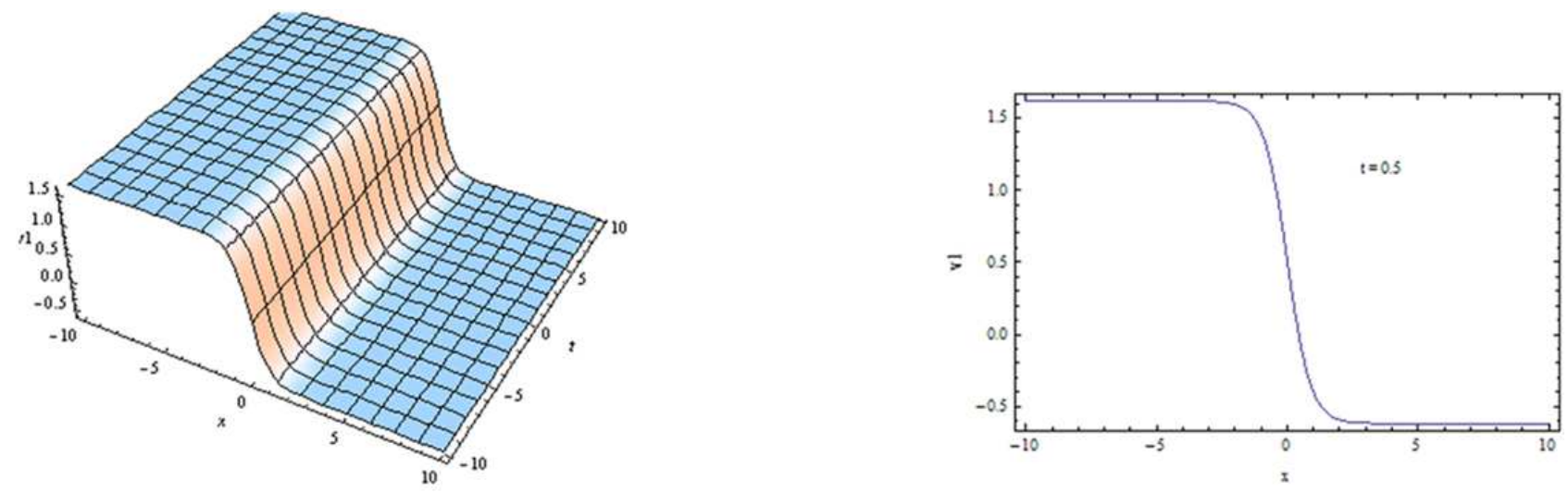

Fig. 5: Three dimensional and two dimensional graphics of the soliton solution of $v(x, t)$ that given by (17) such that $A=2, B=1, C=0.5, k=0.01, c_{1}=c_{2}=0.1, b_{1}=0.5$. 

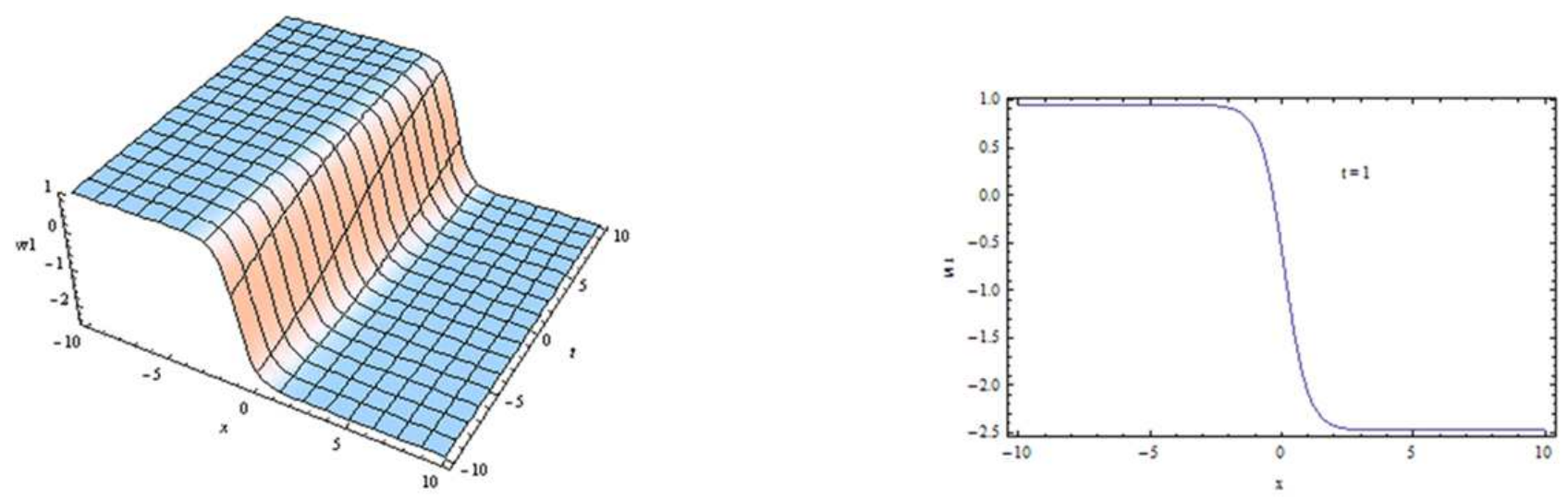

Fig. 6: Three dimensional and two dimensional graphics of the soliton solution of $w(x, t)$ that given by (18) such that $A=2, B=1, C=0.5, k=0.1, c_{1}=c_{2}=0.1$.

\section{Conclusion}

we used the improved $\left(\frac{G^{I}}{G}\right)$ - expansion method for acquiring several new exact solutions for the HSCKdV equation. We have acquired different types solutions which are denoted in terms of trigonometric, triangular, algebric, periodic wave and singular soliton solutions. Some of our reached solutions are new as our research from literature. Consequently, the improved $\left(\frac{G^{I}}{G}\right)$-expansion method is crucial one to construct different types of the soliton solutions of the NPDEs and systems. In addition, this system with time-dependent coefficient and the stochactic perturbation terms will be reported in next works. Additionally, the numerical results that are obtained in this study are in conjuction with the analytical development here (see Figures 1-6).

\section{Competing interests}

The authors declare that they have no competing interests.

\section{Authors' contributions}

All authors have contributed to all parts of the article. All authors read and approved the final manuscript.

\section{References}

[1] Jawad, A. J. M.,Johnson S, Yildirim, A., Kumar, S., Biswas, A., 2013. Soliton solutions to coupled nonlinear wave equations in (2 + 1)-dimensions, Indian J Phys 87: 281.

[2] Wang, M.L., Li, X.Z., Zhang, J.L., 2008. The $\left(\frac{G^{I}}{G}\right)$-expansion method and travelling wave solutions of nonlinear evolution equations in mathematical physics, Phys. Lett. A 372, 417-423.

[3] Bhrawy, A. H., Abdelkawy, M. A.,Biswas, A., 2013. Topological solitons and cnoidal waves to a few nonlinear wave equations in theoretical physics, Indian J. Phys. 87, 1125-1131.

[4] Biswas, A.., Krishnan, E.V.,2011. Exact solutions for Ostrovsky equation, Indian J Phys 85, 1513-1521.

[5] Ray, S.S.,Shaoo, S.,2015. A Novel Analytical Method with Fractional Complex Transform for New Exact Solutions of TimeFractional Fifth-Order Sawada-Kotera Equation, Rep. Math. Phys.75,63-72.

[6] Wu, X. H.,He., J. H.,2007. Solitary solutions, periodic solutions and compacton-like solutions using the Exp-function method, Comput. Math. Appl. 54 966-986. 
[7] Liu, X.,Zhang, W., Li, Z., 2012. Application of Improved (G'/G)-Expansion Method to Traveling Wave Solutions of Two Nonlinear Evolution Equations, Adv. App. math. Mech. 4, 122-130.

[8] Hashemi, M.S., Abbasbandy, S., Alhuthali, M.S. and Alsulami, H.H., 2015. Conservation laws and symmetries of mKdV-KP equation, Romanian Journal of Physics 60 904-917.

[9] Hashemi, M.S. and Wang, G.W., 2017. Lie symmetry analysis and soliton solutions of time-fractional K(m,n) equation, Pramana - J. Phys. 88: 7.

[10] Hashemi, M.S., 2016. On Black-Scholes equation; method of Heir-equations, nonlinear self-adjointness and conservation laws." Bulletin of the Iranian Mathematical Society 42 903-921.

[11] Wu, Y.,Geng, X., Hu, X.,Zhu, S., 1999. A generalized Hirota-Satsuma coupled Korteweg-de Vries equation and Miura transformations, Phys. Lett. A $255,259-264$.

[12] Hirota, R., Satsuma, J.,1981. Soliton solutions of a coupled Korteweg-de Vries equation, Phys. Lett. A 85, 407-408.

[13] Guo-Zhong, Z.,Xi-Jun, Y.,Yun, X.,Jiang, Z.,Di, W.,2010. Approximate analytic solutions for a generalized Hirota-Satsuma coupled KdV equation and a coupled mKdV equation, Chinese Phys. B 19.

[14] Kangalgil, F., Ayaz, F., 2010, Solitary Wave Solutions for HSCKdV Equation and Coupled mKdV Equation by DTM, Arabian J. Sci. Eng., 35(2D), 203-213.,

[15] Liu, J.,Li, H.,2013. Approximate Analytic Solutions of Time-Fractional Hirota-Satsuma Coupled KdV Equation and Coupled MKdV Equation, Abstract and Applied Analysis 11.

[16] Raslan, K.R., 2004. The decomposition method for a Hirota-Satsuma coupled KdV equation and a coupled MKdV equation, Appl. Math. Comput. 81, 1497-1505.

[17] Abbasbandy, S., 2007. The application of homotopy analysis method to solve a generalized Hirota-Satsuma coupled KdV equation, Physics Letters A, 361, 6, 478-483.

[18] Lu, D., Hong, B., Tian, L., 2007, New Explicit Exact Solutions for The Generalized Coupled Hirota-Satsuma KdV System, Comp. Math. Applic., 53, 1181-1190.

[19] Wen, C., Zheng, B., 2013. A New Fractional Sub-equation Method For Fractional Partial Differential Equations, WSEAS Transactions on Math.12,564.

[20] Yong, C., Yan, Z.,Li, B., Zhang, H., 2003.New explicit exact solutions for a generalized Hirota-Satsuma coupled KdV system and a coupled mKdV Equation, Chin. Phys.,12,1-10.

[21] Feng, D., Kezan, L., 2011, Exact traveling wave solutions for a generalized Hirota-Satsuma coupled KdV equation by Fan subequation method, Phys. Lett., A 375, 2201-2210.

[22] Feng, D., Zhang, H., 2008, A new auxiliary function method for solving the generalized coupled Hirota-Satsuma KdV system, Appl. Math. Comput., 200, 283-288.

[23] Yan, Z., 2003, The extended Jacobian elliptic function expansion method and its application in the generalized Hirota-Satsuma coupled KdV system, Chaos, Solitons and Fractals, 15, 575-583. 